\title{
Coexistence of itinerant ferromagnetism and a non-unitary superconducting state with line nodes: possible application to $\mathrm{UGe}_{2}$
}

\author{
Jacob Linder, ${ }^{1}$ Iver B. Sperstad, ${ }^{1}$ Andriy H. Nevidomskyy, ${ }^{2}$ Mario Cuoco, ${ }^{3,4}$ and Asle Sudb $\varnothing^{1}$ \\ ${ }^{1}$ Department of Physics, Norwegian University of Science and Technology, N-7491 Trondheim, Norway. \\ ${ }^{2}$ Department of Physics and Astronomy, Rutgers University, Piscataway, N. J., 08854-8019 \\ ${ }^{3}$ Laboratorio Regionale SuperMat, CNR-INFM Salerno, Baronissi (Sa), Italy \\ ${ }^{4}$ Dipartimento di Fisica E.R. Caianiello, Universita di Salerno, Baronissi(Sa),Italy
}

(Dated: Received November 21, 2018)

\begin{abstract}
We construct a mean-field theory for itinerant ferromagnetism coexisting with a non-unitary superconducting state, where only the majority-spin band is gapped and contains line nodes, while the minority-spin band is gapless at the Fermi level. Our study is motivated by recent experimental results indicating that this may be the physical situation realized in the heavy-fermion compound $\mathrm{UGe}_{2}$. We investigate the stability of the mean-field solution of the magnetic and superconducting order parameters. Also, we provide theoretical predictions for experimentally measurable properties of such a non-unitary superconductor: the specific heat capacity, the Knight shift, and the tunneling conductance spectra. Our study should be useful for direct comparison with experimental results and also for further predictions of the physics that may be expected in ferromagnetic superconductors.
\end{abstract}

PACS numbers: 74.20.-z, 74.25.-q, 74.45.+c, 74.50.+r, 74.20.Rp

\section{INTRODUCTION}

The interplay between ferromagnetic (FM) and superconducting (SC) long range order microscopically coexisting in the same material has attracted much interest during the last decade due to the discovery of superconductivity in ferromagnetic metals, $\mathrm{UGe}_{2}$, URhGe, $\mathrm{UCoGe}$, and possibly $\mathrm{ZrZn}_{2}$

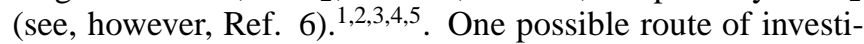
gation of such systems was adopted in early works $\stackrel{7,8,9}{\text {, which }}$ assumed a conventional $s$-wave superconducting condensate residing in a ferromagnetic background caused by localized spins or aligned magnetic impurities. It was shown that below a critical value of the magnetic coupling, comparable to the superconducting gap $\Delta$ itself, superconductivity and magnetism were able to coexist. It was also suggested that a finite momentum pairing state, known as FFLO phase ${ }^{10}$, can appear in the presence of external magnetic field or intrinsic ferromagnetic order, and could thereby permit a larger threshold of the spin exchange energy to coexist with superconductivity.

On the other hand, it has been known since the early days of research on ${ }^{3} \mathrm{He}$ that alternative superconducting states, other than $s$-wave, can be favoured in a ferromagnetic background. The early theories of an equivalent phenomenon to occur in the solid-state have been formulated in the early $1980 \mathrm{~s}^{11}$, despite the absence of any experimental example of a ferromagnetic superconductor at the time $\frac{12}{2}$. With the discovery of superconductivity in $\mathrm{UGe}_{2}$ and $\mathrm{ZrZn}_{2}$, especially given that the same electrons are believed to participate both in ferromagnetism and SC, this latter scenario had to be taken seriously to explain the microscopic coexistence between the two phases. In particular, the very large hyperfine magnetic molecular field in these materials, measured ${ }^{13}$ e.g. with Mössbauer spectroscopy, far exceeds the Pauli limit. This excludes any possibility of singlet-pairing superconductivity.

We should note that although the latter statement is true in $\mathrm{UGe}_{2}$ and other ferromagnetic superconductors, one may still ask whether in principle a singlet-type superconductivity can coexist with ferromagnetism. Although some early theoretical studies $\underline{14}$ indicated that the answer to this question may be affirmative provided FM is weak, a more careful analysis concluded $^{15}$ that the coexistence state of spin-singlet pairing and ferromagnetism always turns out to be energetically unfavorable against the non-magnetic superconducting state even if a finite-momentum pairing (FFLO) state is considered. Later, it was proposed ${ }^{16}$ that the coexistence of metallic ferromagnetism and singlet superconductivity may be realized assuming that the magnetic instability is due to kinetic exchange. However, the coexistence of magnetism and spin-triplet superconductivity appears to be a more promising scenario, since the Cooper pairs may use their spin degree of freedom to align themselves with the internal magnetic field.

An experimental fact that is even more striking is that in all ferromagnetic superconductors known to date, the SC phase is only observed in a small part of the phase diagram otherwise occupied by ferromagnetism $\frac{17}{}$, and it is the region where the magnetism appears to be at its weakest that SC sets in - on the boundary with paramagnetism when the Curie temperature is driven to zero (typically by applying pressure). This immediately raises the question of the microscopic origin of SC pairing, and whether ferromagnetic spin fluctuations play the role of a "glue" for Cooper pair formation very much as they do in superfluid ${ }^{3} \mathrm{He}$. It is equally interesting what role the zero-temperature pressure-tuned phase transition plays in formation of superconductivity and whether notions involving quantum criticality (provided the phase transitions are second order) are necessary to explain the phenomenon.

Although there is no universal answer to this question yet and the research efforts, both experimental and theoretical, are focused on this issue, it is interesting to note that in both $\mathrm{UGe}_{2}$ and $\mathrm{ZrZn}_{2}$ the ferromagnetic phase transition as a function of pressure becomes 1st order as the "critical pressure" is approached at $T=0$. One cannot therefore straightforwardly apply a theory of quantum criticality (be it the Hertz-Millis ${ }^{18}$ theory or one of its variations) given the absence of the quantum critical point as such. It is undeniable however that the 
point where Curie temperature goes to zero is of crucial importance to the formation of the SC state.

Drawing further parallels between triplet-pairing FM superconductors and the superfluid ${ }^{3} \mathrm{He}$, one may wonder whether different symmetries of the SC gap can occur, as is the case in the different phases $\frac{19}{19}$ of ${ }^{3} \mathrm{He}$. For example, can the gap symmetry with point or line nodes be realized in the ferromagnetic superconductors? Very recently, experimental evidence has appeared which suggests that the answer is 'yes'. Harada et $a l .{ }^{20}$ reported on ${ }^{73} \mathrm{Ge}$ nuclear-quadrupole-resonance experiments performed under pressure, in which the nuclear spinlattice relaxation rate revealed an unconventional nature of superconductivity implying that the majority spin band in $\mathrm{UGe}_{2}$ was gapped with line nodes, while the minority spin band remained gapless at the Fermi level.

Motivated by this, we present a mean-field model for coexisting ferromagnetism and spin-triplet superconductivity with a SC order parameter that displays line nodes in majorityspin channel and is gapless for minority spin. We first study the interplay between the magnetic and superconducting order parameters, and then proceed to make several predictions for experimentally relevant quantities: the specific heat capacity, Knight shift, and tunneling conductance. Let us briefly summarize our main results. We find that the low-temperature specific heat capacity $C_{V}$ shows power-lawer behaviour (to be contrasted with the conventional exponential decay in the $s$-wave case), and that the gapless minority spins dominate the contribution to $C_{V}$ at low temperatures, giving rise to a linear $T$-dependence. Also, the relative jump in $C_{V}$ shows a strong dependence on the exchange splitting in the system. With regard to the Knight shift, we find that it is suppressed at $T=0$ with increasing exchange splitting of the majority and minority spin bands when the external field is applied perpendicular to the spin of the Cooper pairs in the system. In general, however, it depends strongly on the orientation of the field with respect to the crystallographic axes of the compound, indicative of the triplet pairing in the system. Finally, the normalized tunneling conductance spectra show a strong directional dependence with respect to the orientation of the superconducting order parameter in reciprocal space, but change very little upon modifying the exchange splitting in the system. Our findings should be useful for comparison with experimental studies, and could lead to further insights as regards the nature of the superconducting order parameter.

This paper is organized as follows. We first describe the phenomenological framework to be used in this work in Sec. [II We then present our theoretical model in Sec. [III] and provide the results of the self-consistent mean-field treatment at both zero and finite temperatures in Sec. IVA We then proceed to make predictions for experimentally accessible quantities in Sec. IV B] using the self-consistently obtained results from Sec. IVA. We discuss our findings in Sec. VI, and summarize in Sec. VII] We will use boldface notation for vectors, $\therefore$ for operators, $\ldots$ for $2 \times 2$ matrices, and $\hat{\ldots}$ for $4 \times 4$ matrices.

\section{PHENOMENOLOGICAL FRAMEWORK}

The issue of coexisting ferromagnetism and superconductivity dates back to half a century ago when the celebrated FFLO state was predicted $^{10}$ as a finite-momentum pairing state with real-space structure of the singlet SC order parameter that may develop under certain conditions close to the critical magnetic field $H_{c 2}$. The conditions for the FFLO state are however very different from those observed in ferromagnetic superconductors such as $\mathrm{UGe}_{2}$. In particular, as has already been emphasised above, the magnetic molecular field felt by Cooper pairs inside the ferromagnet is many times larger ${ }^{13}$ than the Pauli limiting field necessary to destroy the singlet Cooper pairs. We shall therefore concentrate on triplet-type superconducting pairing.

Several remarks are in order. We note from the outset that the ferromagnetism observed in the Uranium compounds and in $\mathrm{ZrZn}_{2}$ is itinerant, Stoner-like in its nature. We shall therefore not discuss the topic of localized magnetic moments that would have, among other things, provided a pair-breaking mechanism in accord with Abrikosov-Gor'kov theory ${ }^{21}$ of magnetic scattering. Here, we will assume that the same electrons involved in the spontaneous SU(2) symmetry breaking associated with ferromagnetism, also participate in the U(1) gauge symmetry breaking that characterizes a superconductor.

The idea of triplet pairing occurring between the same electrons that form the Stoner instability at the border of ferromagnetism goes back to Fay and Appe1 ${ }^{11}$ (1980) who considered exchange of magnetic spin fluctuations as a microscopic mechanism for Cooper pairing. More recently, the problem has been revisited $22,23,24,25,26$ in the light of experimental findings in $\mathrm{UGe}_{2}$ and other ferromagnetic superconductors.

In this paper, we shall take a phenomenological approach to superconductivity, leaving the intriguing and debated question of the microscopic mechanism for Cooper pairing aside. In particular, we shall consider systems where superconductivity appears at a lower temperature than the temperature at which onset of ferromagnetism is found. This is certainly the case experimentally and may be simply due to the fact that the energy scales for the two phenomena are quite different, with the exchange energy naturally being the largest. It may, however, also be due to the fact that superconductivity is dependent on ferromagnetism for its very existence. Such a suggestion has recently been put forth ${ }^{32}$.

A crucial issue to address in this context is whether superconductivity and ferromagnetism are phase-separated (such as e.g. solid and liquid phases coexisting at the melting point) or not. Fairly strong experimental evidence for non-phaseseparated coexistence of ferromagnetism and superconductivity has recently been presented in $\mathrm{UGe}_{2}{ }^{33}$. However, even if such non-phase-separated coexistence is established, there still remains the issue of whether the superconducting orderparameters exhibits spatial variations, precisely due to its nonphase-separated coexistence with ferromagnetic order. One obvious candidate for such spatial variations 34 is a spontaneously formed Abrikosov vortex lattice, induced by the internal magnetization M. As argued in Ref. 35, an impor- 
tant factor with respect to whether a vortex lattice appears or not could be the magnitude of the internal magnetization M. Specifically, Ref. 36 suggested that vortices may arise if $4 \pi \mathbf{M}>\mathbf{H}_{c 1}$, where $\mathbf{H}_{c 1}$ is the lower critical field. It is conceivable that a weak ferromagnetic state coexisting with superconductivity may give rise to a domain structure, in the absence of an external field, that is vortex-free. Therefore, we shall consider non-phase-separated coexistence of the FM and $\mathrm{SC}$ order parameters from here on, as have other studies ${ }^{37}$. We will also leave the complications arising from the spatial variation of the superconducting order parameter originating with a putative spontaneously formed Abrikosov vortex lattice in the superconducting order parameter for future investigations.

Spin-triplet superconductors are characterized by a multicomponent order parameter, which for the simplest case of the $p$-wave may be expressed in terms of three independent components of a $\mathbf{d}$-vector:

$$
\mathbf{d}_{\mathbf{k}}=\left[\frac{\Delta_{\mathbf{k} \downarrow \downarrow}-\Delta_{\mathbf{k} \uparrow \uparrow}}{2}, \frac{-1\left(\Delta_{\mathbf{k} \downarrow \downarrow}+\Delta_{\mathbf{k} \uparrow \uparrow}\right)}{2}, \Delta_{\mathbf{k} \uparrow \downarrow}\right] .
$$

Note that $\mathbf{d}_{\mathbf{k}}$ transforms like a vector under spin rotations. In terms of the components of $\mathbf{d}_{\mathbf{k}}$, the order parameter itself is a $2 \times 2$ matrix that reads

$$
\check{\Delta}_{\alpha \beta}(\mathbf{k}) \equiv\left\langle c_{\mathbf{k}, \alpha} c_{\mathbf{k}, \beta}\right\rangle=\left[i\left(\mathbf{d}_{\mathbf{k}} \cdot \boldsymbol{\sigma}\right) \sigma_{y}\right]_{\alpha \beta},
$$

where $\boldsymbol{\sigma}$ is the vector of Pauli matrices, and $c_{\mathbf{k}, \alpha}^{\dagger}, c_{\mathbf{k}, \alpha}$ are the usual electron creation-annihilation operators for momentum $\mathbf{k}$ and $\operatorname{spin} \alpha$.

The superconducting order parameter is characterized as unitary if the modulus of the gap is proportional to the unity matrix: $\left(\check{\Delta} \cdot \check{\Delta}^{\dagger}\right) \propto \check{1}$. Written in terms of the vector $\mathbf{d}_{\mathbf{k}}$, this condition is equivalent to the requirement that $\left\langle\mathbf{S}_{\mathbf{k}}\right\rangle=0$, where we have introduced the net magnetic moment (or spin) of the Cooper pair

$$
\left\langle\mathbf{S}_{\mathbf{k}}\right\rangle \equiv 1\left(\mathbf{d}_{\mathbf{k}} \times \mathbf{d}_{\mathbf{k}}^{*}\right) .
$$

The unitary triplet state thus has Cooper pairs with zero magnetic moment, whereas the non-unitary state is characterized by non-zero value of $\left\langle\mathbf{S}_{\mathbf{k}}\right\rangle \neq 0$. The latter effectively means that time-reversal symmetry is spontaneously broken in the spin part of the Cooper pairs 27 . It is thus intuitively clear that having the spin of the Cooper pair aligned with the internal magnetic field of the ferromagnet can lower the energy of the resulting coexistence state. The above argument that the order parameter in the ferromagnetic superconductors must be nonunitary has been put forward by Machida and $\mathrm{Ohmi}^{23}$, and others 24,28 . Distinguishing between unitary and non-unitary states in ferromagnetic superconductors is clearly one of the primary objectives in terms of identifying the correct SC order parameter. To this end, recent studies have focused on calculating transport properties of ferromagnetic superconductors $40,41,42,43,44,45$. There have also been investigations of identifying spin-triplet pairing in quasi-1D materials ${ }^{46,47,48,49}$.

Finally, we note that inter-subband pairing is expected to be strongly suppressed in the presence of the Zeeman splitting between the $\uparrow, \downarrow$ conduction sub-bands. In other words, only electrons within the same subband will form Cooper pairs (the so-called equal-spin pairing) and we shall set $\Delta_{\uparrow \downarrow}=0$ in what follows. Moreover, the requirement of non-unitarity of the order parameter then reduces to the requirement that the vector $\mathbf{d}_{\mathbf{k}}$ in Eq. (1) should have two non-zero components, $i$. e. $\Delta_{\uparrow \uparrow} \neq \Delta_{\downarrow \downarrow}$, which one would expect anyway in the presence of the Zeeman splitting between the two spin subbands. The spin of the Cooper pair is then $\left\langle S_{z}\right\rangle=\frac{1}{2}\left(\left|\Delta_{\uparrow \uparrow}\right|^{2}-\left|\Delta_{\downarrow \downarrow}\right|^{2}\right)$ and is aligned along the magnetic field ( $z$ being the spin quantization axis).

\section{THEORY}

We consider a model of a ferromagnetic superconductor described by uniformly coexisting itinerant ferromagnetism and non-unitary, spin-triplet superconductivity. We write down a weak-coupling mean-field theory Hamiltonian with equalspin pairing Cooper pairs and a finite magnetization along the easy-axis similar to the model studied in Refs. 25.26, namely

$$
\begin{aligned}
\hat{H} & =\sum_{\mathbf{k}} \xi_{\mathbf{k}}+\frac{I N M^{2}}{2}-\frac{1}{2} \sum_{\mathbf{k} \sigma} \Delta_{\mathbf{k} \sigma \sigma}^{\dagger} b_{\mathbf{k} \sigma \sigma} \\
& +\frac{1}{2} \sum_{\mathbf{k} \sigma}\left(\hat{c}_{\mathbf{k} \sigma}^{\dagger} \hat{c}_{-\mathbf{k} \sigma}\right)\left(\begin{array}{cc}
\xi_{\mathbf{k} \sigma} & \Delta_{\mathbf{k} \sigma \sigma} \\
\Delta_{\mathbf{k} \sigma \sigma}^{\dagger} & -\xi_{\mathbf{k} \sigma}
\end{array}\right)\left(\begin{array}{c}
\hat{c}_{\mathbf{k} \sigma} \\
\hat{c}_{-\mathbf{k} \sigma}^{\dagger}
\end{array}\right),
\end{aligned}
$$

where $b_{\mathbf{k} \sigma \sigma}=\left\langle c_{-\mathbf{k} \sigma} c_{\mathbf{k} \sigma}\right\rangle$ is the non-zero expectation value of the pair of Bloch states. Applying a standard diagonalization procedure, we arrive at

$$
\begin{aligned}
\hat{H} & =H_{0}+\sum_{\mathbf{k} \sigma} E_{\mathbf{k} \sigma} \hat{\gamma}_{\mathbf{k} \sigma}^{\dagger} \hat{\gamma}_{\mathbf{k} \sigma}, \\
H_{0} & =\frac{1}{2} \sum_{\mathbf{k} \sigma}\left(\xi_{\mathbf{k} \sigma}-E_{\mathbf{k} \sigma}-\Delta_{\mathbf{k} \sigma \sigma}^{\dagger} b_{\mathbf{k} \sigma \sigma}\right)+\frac{I N M^{2}}{2},
\end{aligned}
$$

where $\left\{\hat{\gamma}_{\mathbf{k} \sigma}, \hat{\gamma}_{\mathbf{k} \sigma}^{\dagger}\right\}$ are new fermion operators and the eigenvalues read

$$
E_{\mathbf{k} \sigma}=\sqrt{\xi_{\mathbf{k} \sigma}^{2}+\left|\Delta_{\mathbf{k} \sigma \sigma}\right|^{2}}
$$

It is implicit in our notation that $\xi_{\mathbf{k}}=\varepsilon_{\mathbf{k}}-E_{F}$ is measured from the Fermi level, where $\varepsilon_{\mathbf{k}}$ is the kinetic energy. The free energy is obtained through

$$
F=H_{0}-\frac{1}{\beta} \sum_{\mathbf{k} \sigma} \ln \left(1+\mathrm{e}^{-\beta E_{\mathbf{k} \sigma}}\right),
$$

such that the gap equations for the magnetic and superconducting order parameters become ${ }^{25}$

$$
\begin{aligned}
M & =-\frac{1}{N} \sum_{\mathbf{k} \sigma} \frac{\sigma \xi_{\mathbf{k} \sigma}}{2 E_{\mathbf{k} \sigma}} \tanh \left(\beta E_{\mathbf{k} \sigma} / 2\right), \\
\Delta_{\mathbf{k} \sigma \sigma} & =-\frac{1}{N} \sum_{\mathbf{k}^{\prime}} V_{\mathbf{k}^{\prime} \sigma \sigma} \frac{\Delta_{\mathbf{k}^{\prime} \sigma \sigma}}{2 E_{\mathbf{k}^{\prime} \sigma}} \tanh \left(\beta E_{\mathbf{k}^{\prime} \sigma} / 2\right) .
\end{aligned}
$$

Specifically, we now consider a model which should be of relevance to the ferromagnetic superconductor $\mathrm{UGe}_{2}$, and possibly also for UCoGe and URhGe. In Ref. 20, it was argued 
that the majority spin (spin-up in our notations) fermions were gapped and that the order parameter displayed line nodes, while the minority (spin-down) fermions remained gapless at the Fermi level in the heavy-fermion compound $\mathrm{UGe}_{2}$. An obvious mechanism for suppressing the superconducting instability in the minority-spin channel as compared to the majority-spin channel is the difference in density of states (DOS) at the Fermi level. Indeed, from Fig. 1 in Ref. 25 (see also Fig. 4 in Ref. 26), it is seen that the critical temperature for pairing in the minority-spin subband, $T_{c}^{\downarrow}$, is predicted to be much smaller than the critical $T_{c}^{\uparrow}$ for the majorityspin subband, even for quite weak magnetic exchange splittings. Given the already quite low critical temperature $T_{c}$ that is observed experimentally in ferromagnetic superconductors $\left(T_{c} \lesssim 1 \mathrm{~K}\right)$, which we associate with $T_{c}^{\uparrow}$, we therefore conclude that it might indeed be very hard to observe experimentally the even smaller gap in the minority-spin subband. Therefore, it is permissible to only consider pairing in the majority-spin channel and neglect a small (if any) pairing between minority-spin electrons. In our notation this means setting $M \neq 0, \Delta_{\mathbf{k} \uparrow} \neq 0, \Delta_{\mathbf{k} \downarrow}=0$.

We stress that the above statement, although intuitively attractive, may need further justification since we have so far neglected completely the spin-orbit interaction that is expected to be strong in Urainium based compounds, such as $\mathrm{UGe}_{2}$, URhGe and UCoGe. The effect of the latter would be to provide some effective coupling between majority and minority spin subbands and would probably lead to induced SC order parameter in minority spin channel. This issue is left for future study ${ }^{50}$.

To model the presence of line nodes in the order parameter, we choose

$$
\Delta_{\mathbf{k} \uparrow}=\Delta_{\overline{\mathbf{k}}_{F} \uparrow \uparrow}=\Delta_{0} \cos \theta,
$$

where $\overline{\mathbf{k}}_{F}$ is the normalized Fermi wave-vector, such that the gap only depends on the direction of the latter. This is the weak-coupling approximation. The above gap satisfies the correct symmetry requirement dictated by the Pauli principle, namely a sign change under inversion of momentum, $\theta \rightarrow \pi-\theta$. Here, $\theta$ is the azimuthal angle in the $x y$-plane. Our choice of this particular symmetry for the $p$-wave superconducting gap is motivated by the experimental results of Harada et al. ${ }^{20}$. The $\cos \theta$-dependence is also in accord with the results of Ref. 30 , which showed that the majority band at the Fermi level for $\mathrm{UGe}_{2}$ is strongly anisotropic with a small dispersion along the $k_{y}$-direction. We consider here a situation where the electrons are restricted from moving along the $\mathbf{z}$-axis. The motivation for this is that, strictly speaking, it seems plausible that uniform coexistence of ferromagnetic and superconducting order should only be realized in thin-film structures where the Meissner (diamagnetic) response of the superconductor is suppressed for in-plane magnetic fields. The thin-film structure would then also suppress the orbital effect of the field. In a bulk structure, as considered in Ref. 14, we expect that a spontaneous vortex lattice should be the favored thermodynamical state ${ }^{34}$, unless prohibited by a possible domain structure. Having said that, we point out that there is no firm experimental evidence for the presence of such a vortex phase in ferromagnetic superconductors such as $\mathrm{UGe}_{2}$ and $\mathrm{ZrZn}_{2}$, and we therefore do not exclude some mechanism that would instead stabilise a truly uniform coexistence of the SC and FM in these materials. It should be mentioned that uniform coexistence of ferromagnetism and superconducting order have also been speculated to occur in quasi-1D and quasi-2D materials such as $\mathrm{RuSr}_{2} \mathrm{GdCu}_{2} \mathrm{O}_{8}$. In our model, the pairing potential may be written as

$$
V\left(\theta, \theta^{\prime}\right)=-g \cos \theta \cos \theta^{\prime}
$$

where $g$ is the weak-coupling constant. Conversion to integral equations is accomplished by means of the identity

$$
\frac{1}{N} \sum_{\mathbf{k}} f\left(\xi_{\mathbf{k} \sigma}\right)=\int \mathrm{d} \varepsilon N^{\sigma}(\varepsilon),
$$

where $N^{\sigma}(\varepsilon)$ is the spin-resolved density of states. In three spatial dimensions, this may be calculated from the dispersion relation by using the formula

$$
N^{\sigma}(\varepsilon)=\frac{V}{(2 \pi)^{3}} \int_{\varepsilon_{\mathbf{k} \sigma}=\text { const }} \frac{\mathrm{d} S_{\varepsilon_{\mathbf{k} \sigma}}}{\left|\hat{\nabla}_{\mathbf{k}} \varepsilon_{\mathbf{k} \sigma}\right|} .
$$

With the dispersion relation $\xi_{\mathbf{k} \sigma}=\varepsilon_{\mathbf{k}}-\sigma I M-E_{F}$, one obtains

$$
N^{\sigma}(\varepsilon)=\frac{m V \sqrt{2 m\left(\varepsilon+\sigma I M+E_{F}\right)}}{2 \pi^{2}} .
$$

In their integral form, Eqs. (8) for the order parameters read

$$
\begin{aligned}
M=- & \frac{1}{4 \pi} \sum_{\sigma} \sigma \int_{0}^{2 \pi} \int_{-E_{F}-\sigma I M}^{\infty} \mathrm{d} \theta \mathrm{d} \varepsilon \frac{\varepsilon N^{\sigma}(\varepsilon)}{E_{\sigma}(\varepsilon, \theta)} \\
& \times \tanh \left[\beta E_{\sigma}(\varepsilon, \theta) / 2\right], \\
1= & \frac{g}{4 \pi} \int_{0}^{2 \pi} \int_{-\omega_{0}}^{\omega_{0}} \mathrm{~d} \theta \mathrm{d} \varepsilon \frac{N^{\uparrow}(\varepsilon) \cos ^{2} \theta}{E_{\uparrow}(\varepsilon)} \tanh \left[\beta E_{\uparrow}(\varepsilon, \theta) / 2\right] .
\end{aligned}
$$

For ease of notation, we also define

$$
\begin{aligned}
\Delta_{\sigma}(\theta) & =\left\{\begin{array}{ll}
\Delta_{0} \cos \theta & \text { if } \sigma=\uparrow \\
0 & \text { if } \sigma=\downarrow
\end{array}\right\}, \\
E_{\sigma}(\varepsilon, \theta) & =\left\{\begin{array}{ll}
\sqrt{\varepsilon^{2}+\Delta_{0}^{2} \cos ^{2} \theta}, & \text { if } \sigma=\uparrow \\
\varepsilon & \text { if } \sigma=\downarrow
\end{array}\right\} .
\end{aligned}
$$

For the following treatment, we define $\tilde{M}=I M / E_{F}$, i.e. the exchange energy scaled on the Fermi energy. Moreover, we set $c=g N(0) / 2$ to a typical value of 0.2 and $\tilde{\omega}_{0}=\omega_{0} / E_{F}=$ 0.01 as the typical spectral width of the bosons responsible for the attractive pairing potential. Finally, we define the parameter $\tilde{I}=I N(0)$ as a measure of the magnetic exchange coupling. As discussed below, only for $\tilde{I}>1$ will a spontaneous magnetization appear in our model, in agreement with the Stoner criterion for itinerant ferromagnetism. 


\section{RESULTS: MEAN-FIELD MODEL FOR COEXISTENCE}

\section{A. Zero temperature case}

For zero-temperature, the superconducting gap equation reads

$$
1=\frac{g}{4 \pi} \int_{0}^{2 \pi} \int_{-\omega_{0}}^{\omega_{0}} \mathrm{~d} \theta \mathrm{d} \varepsilon \frac{N^{\uparrow}(\varepsilon) \cos ^{2} \theta}{\sqrt{\varepsilon^{2}+\Delta_{0}^{2} \cos ^{2} \theta}} .
$$

Under the assumption that $\omega_{0} \gg \Delta_{0}$, we obtain that

$$
\frac{2}{c \sqrt{1+\tilde{M}}}=\ln \left(\frac{2 \omega_{0}}{\Delta_{0}}\right)-\frac{1}{\pi} \int_{0}^{2 \pi} \mathrm{d} \theta \cos ^{2} \theta \ln |\cos \theta| .
$$

which may be solved to yield the zero-temperature gap

$$
\Delta_{0}=2.426 \omega_{0} \exp [-2 /(c \sqrt{1+\tilde{M}})]
$$

By inserting Eq. (18) into the gap equation for the magnetization in Eq. (14), we have managed to decouple the selfconsistency equations for $M$ and $\Delta_{0}$. Numerical evaluation reveals that the gap equation for $M$ is completely unaffected by the presence of $\Delta_{0}$, which physically means that the magnetization remains unaltered with the onset of superconductivity. This is reasonable in a model where the energy scale for the onset of magnetism is vastly different from the energy scale for superconductivity, such that by the time superconductivity sets in, the ordering of the spins essentially exhausts the maximum possible magnetisation.

The dependence of $\Delta_{0}$ on $\tilde{I}$ is shown in Fig. 11. The gap remains constant for $\tilde{I} \in[0,1]$, which is a unitary phase. In the unitary phase, there is no reason for the minority spin band to remain ungapped when $M=0$, and hence we would expect two gaps $\Delta_{\uparrow}=\Delta_{\downarrow}$ of equal magnitude for $\tilde{I}<1$. Our model of gapping exclusively for the majority spin band is therefore justified only for $\tilde{I}>1$, which is the regime we shall be concerned with throughout this article. The onset of a spontaneous magnetization for $\tilde{I}>1$ is the well-known Stoner criterion for an isotropic electron gas, where the spin susceptibility may be written as 52

$$
\begin{aligned}
\chi(\mathbf{q}, \omega) & =\frac{\chi_{0}(\mathbf{q}, \omega)}{1-I \chi_{0}(\mathbf{q}, \omega)}, \\
\chi_{0}(\mathbf{q}, \omega) & =N_{0}\left(1-\frac{\mathbf{q}^{2}}{12 k_{F}^{2}}+1 \frac{\pi \omega}{2 v_{F}|\mathbf{q}|}\right), \\
|\mathbf{q}| & \ll 2 k_{F}, \omega \ll E_{F} .
\end{aligned}
$$

For a parabolic band, the static susceptibility is maximal for $\mathbf{q}=0$ where

$$
\chi(\mathbf{q}=0, \omega=0)=\frac{N_{0}}{1-I N(0)}=\frac{N_{0}}{1-\tilde{I}} .
$$

The introduction of a ferromagnetic order is demarcated by the divergence of the susceptibility for $\tilde{I}=1$, which is precisely Stoner's criterion for itinerant ferromagnetism. In the absence of superconductivity, the self-consistency equation for the magnetization at $T=0$ reduces to

$$
h=-\frac{\tilde{I}}{3 \sqrt{E_{F}}} \sum_{\sigma} \sigma\left[\left(E_{F}+\sigma h+\eta\right)^{3 / 2}-2\left(E_{F}+\sigma h\right)^{3 / 2}\right],
$$

where $\eta$ is an upper energy cut-off determined by the bandwidth and $h=I M$ is the exchange splitting of the majority and minority bands. Since the energy scales for the magnetic and superconducting order parameter differ so greatly in magnitude, Eq. (21) is an excellent approximation even in the coexistent state (we have verified this numerically).

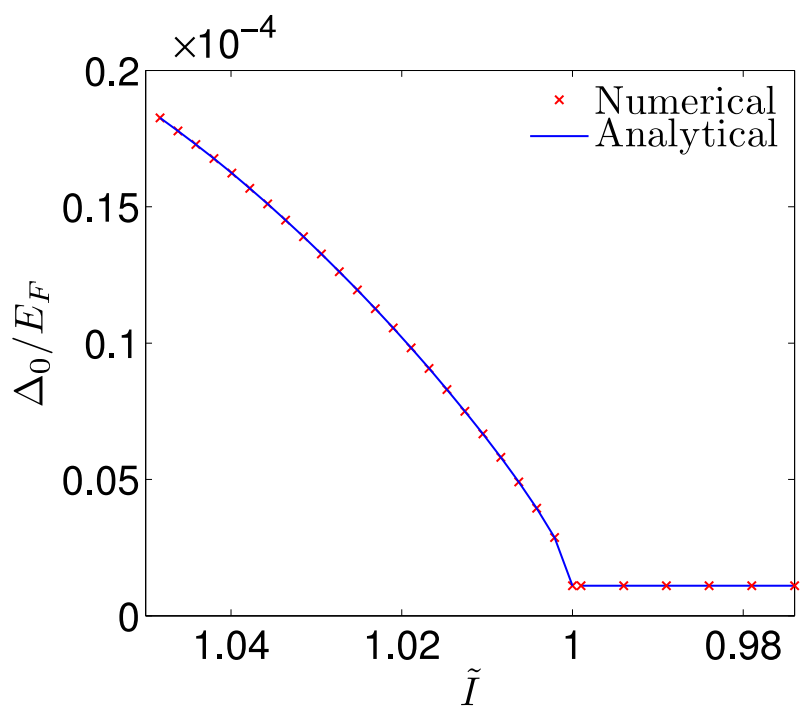

FIG. 1: (Color online) The gap-dependence on the ferromagnetic exchange interaction parameter $\tilde{I}=I N(0)$. The gap remains constant for $\tilde{I} \in[0,1]$, corresponding to a unitary phase. The gap $\Delta_{0}$ then starts growing with increasing $\tilde{I}$ for $\tilde{I}>1$.0, announcing the onset of a spontaneous magnetization. The analytical formula is based on Eq. (18).

\section{B. Finite temperature case}

The critical temperature for the superconducting order parameter is obtained in the standard way [setting $\Delta_{0}=0$ in Eq. [16] ] to yield

$$
T_{c}=1.134 \omega_{0} \exp [-2 /(c \sqrt{1+\tilde{M}})] .
$$

In Fig. 2, we plot the temperature-dependence of the selfconsistently obtained solution of $\Delta_{0}$ and compare it to the analytical mean-field temperature dependence

$$
\Delta_{0}(T)=\Delta_{0}(0) \tanh \left[\gamma \sqrt{T_{c} / T-1}\right]
$$

The BCS result is $\gamma=1.74$, but we find a better fit for our numerical results using $\gamma=1.70$. Throughout the rest of this paper, we shall therefore make use of Eq. (23) with 
$\chi=1.70$ to model the temperature-dependence of the gap for $\tilde{I}=\{1.01,1.02,1.03\}$, since the agreement is excellent with the full numerical solution. As in the zero-temperature case, we find that the gap equations in Eq. (14) may be completely decoupled also at finite temperature. We have verified that the gap equation for the superconducting order parameter has a unique non-trivial solution, which guarantees that the system will prefer to be in the coexistent state of ferromagnetism and superconductivity. The phase-diagram of the model we are

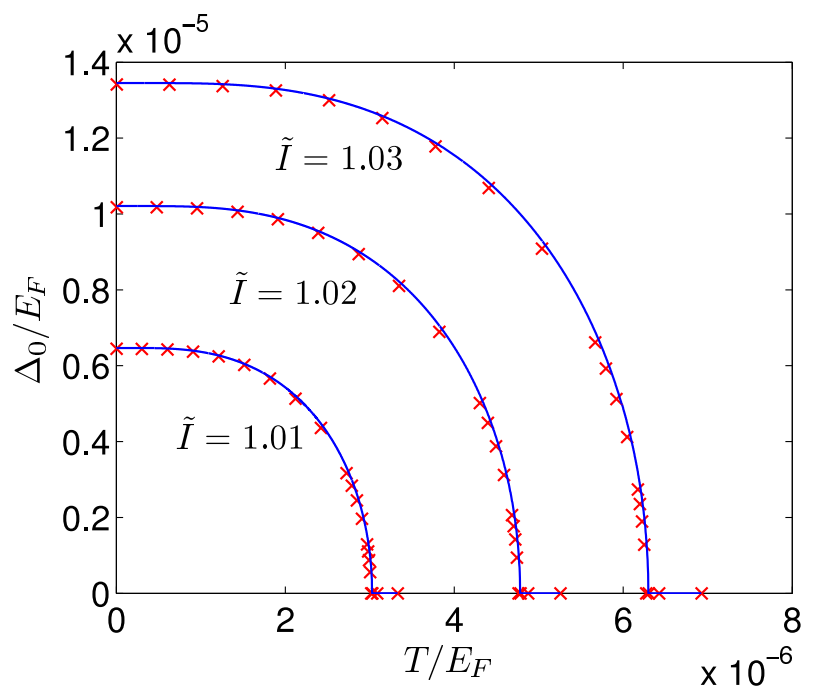

FIG. 2: (Color online) Self-consistently obtained solution for the superconducting gap $\Delta_{0}$ (red symbols) compared to the analytical expression Eq. 23] with $\gamma=1.70$ (blue lines), modelling a BCS-like temperature dependence.

considering may be obtained numerically and is shown in Fig. 3. As seen, a quantum phase transition may occur at $\tilde{I}=1.0$, separating the 'unitary' superconducting state (see discussion in an earlier paragraph) from the ferromagnetic, non-unitary superconducting state. The critical temperature for the magnetic order parameter is orders of magnitudes larger than $T_{c}$ for the superconductivity except for very close to $\tilde{I}=1.0$. The increase in $T_{c}$ in the non-unitary phase as compared to the unitary phase is a result of the increase in density of states with magnetization for the majority spin.

Experimentally, one often maps out the $T$ - $p$ phase diagram, where $T$ is temperature and $p$ is pressure. Note that the value of $\tilde{I}$ may be controlled experimentally by adjusting the pressure on the sample. A change in pressure is accompanied by a change in the width of the electron bands, and therefore directly affects the density of states at the Fermi level: increasing the pressure on the samples reduces the density of states, and hence also the effective coupling constant $\tilde{I} I \underline{55}$. A notable feature in the phase diagram for $\mathrm{UGe}_{2}$ as determined experimentally, is that superconductivity only appears in the ferromagnetic phase, and not in the paramagnetic phase.

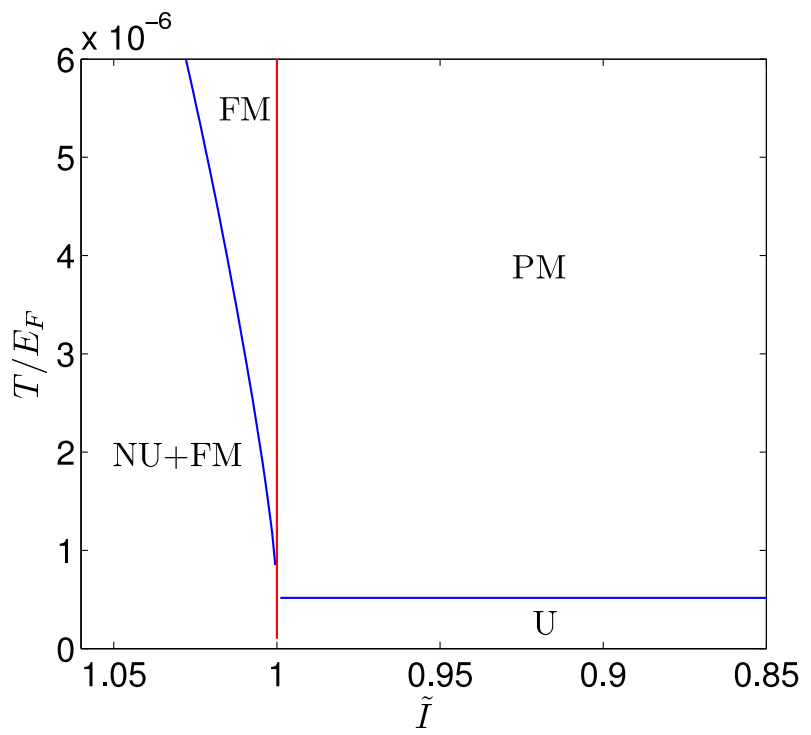

FIG. 3: (Color online) The phase-diagram of our model in the $T-\tilde{I}$ plane. For $\tilde{I}>1.0$, a spontaneous magnetization arises and allows for the possible uniform coexistence of ferromagnetism and triplet superconductivity. Note that decreasing $\tilde{I}$ (going from left to right along the $\mathrm{x}$-axis) corresponds to an increasing external pressure $p$. The abbrevations stand for non-unitary (NU), unitary (U), ferromagnetic (FM), and paramagnetic (PM).

\section{RESULTS: EXPERIMENTAL PREDICTIONS}

We next proceed to using the self-consistently obtained solutions from the previous section to make predictions for three experimental quantities that are routinely used to study superconducting condensates: specific heat, Knight shift, and tunneling conductance spectra. We first consider the normalized heat capacity, which is defined as

$$
\begin{aligned}
C_{V} & =\frac{\beta^{2}}{8 \pi} \sum_{\sigma} \int_{0}^{2 \pi} \int_{-E_{F}-\sigma I M}^{\infty} \mathrm{d} \theta \mathrm{d} \varepsilon \frac{N^{\sigma}(\varepsilon)}{\cosh ^{2}\left[\beta E_{\sigma}(\varepsilon, \theta) / 2\right]} \\
& \times\left[E_{\sigma}^{2}(\varepsilon, \theta)-T\left(\Delta_{\sigma}(\theta) \frac{\partial \Delta_{\sigma}(\theta)}{\partial T}-\sigma \varepsilon I \frac{\partial M}{\partial T}\right)\right] .
\end{aligned}
$$

Since the critical temperature of $M$ is much larger than the critical temperature for $\Delta_{0}$ in our model, we may safely neglect $\partial M / \partial T$ in the low-temperature regime. Consider Fig. 4 for a plot of the specific heat capacity using three representative values for $\tilde{I}$. The general trend with increasing $\tilde{I}$ is an increase of the jump of $C_{V}$ at $T=T_{c}$. The physical reason for this is that the majority spin carriers will dominate the jump in specific heat stronger when the exchange splitting between the bands increases, which is in agreement with the results of Ref. 26. Analytically, the relative jump in specific heat may be expressed as

$$
\left.\left(\frac{\Delta C_{V}}{C_{V}}\right)\right|_{T=T_{c}} \sim\left(1+\sqrt{\frac{1-h / E_{F}}{1+h / E_{F}}}\right)^{-1} .
$$

It depends on the exchange splitting in the superconductor since the contribution from the majority spin carriers will tend 
to dominate the specific heat when $h$ increases. The lowtemperature scaling with $T$ bears witness of the line nodes in the gap, and is to be contrasted with the more rapidly decaying $s$-wave case. Also note that the minority spin fermions are in the normal state and give a significant contribution to the specific heat in form of a linear $T$-dependence at low temperatures. If both spin species were gapped with line nodes, one would expect a $T^{2}$-dependence of the low temperature specific heat.

In the experimental study of the heat-capacity in $\mathrm{UGe}_{2}$ conducted in Ref. 60, a peak of the heat-capacity associated with the superconducting transition was observed in a narrow pressure region $\Delta p \simeq 0.1 \mathrm{GPa}$ around $p_{x}$. Here, $p_{x}$ is the pressure at which the superconducting transition temperature $T_{c}$ shows a maximum value. Farther away from $p_{x}$, the heat capacity anomaly was smeared out. In particular, a substantial residual value of $C_{V} / T$ was observed at $T \rightarrow 0$. The authors of Ref. 60 argued that neither the minority band density of states at the Fermi level nor the contribution from a self-induced vortex state would be appropriate to describe this residual value. Instead, it might stem from impurities that induce a finite density of states at the Fermi level. For an anisotropic superconductor like $\mathrm{UGe}_{2}$, the residual value would be highly sensitive to such impurities. It is also clear that the observation of sharp peaks, similar to the ones we obtain in Fig. 4, depend strongly on the applied pressure on the superconductor, and in particular to how close it is to $p_{x}$.
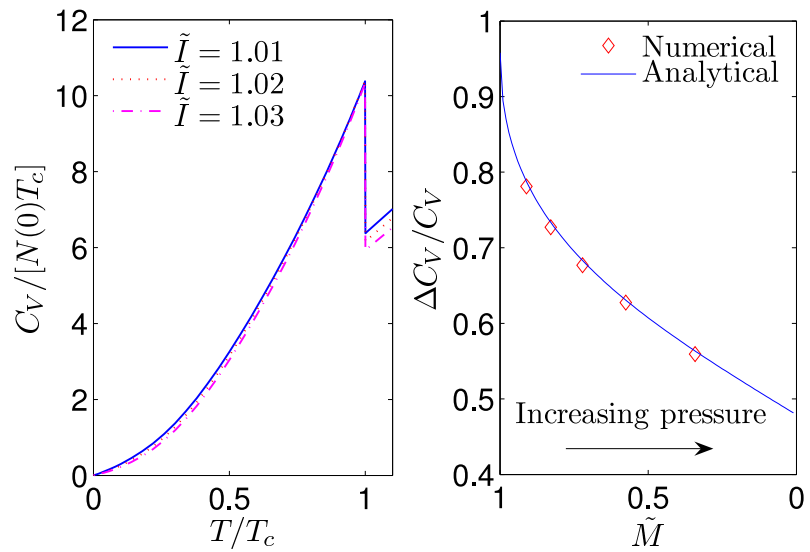

FIG. 4: (Color online) The left panel shows a plot of the specific heat capacity, using self-consistently obtained order parameters, for three different values of $\tilde{I}$. The right panel shows relative jump (superconducting vs. normal state) of the specific heat at the transition temperature as a function of the normalized exchange splitting between the spin bands, $\tilde{M}$. Numerically calculated values are shown in red, analytical result [Eq. [25] using $\gamma \approx 1.70$ are shown in blue.

We next consider the spin susceptibility, making use of the standard formula $a^{31}$

$$
\chi(\mathbf{q}, \omega)=-\frac{1}{2 \beta} \sum_{\mathbf{k}, 1 \omega_{n}} \operatorname{Tr}\left\{\hat{\mathcal{G}}\left(\mathbf{k}, 1 \omega_{n}\right) \hat{\mathcal{G}}\left(\mathbf{k}+\mathbf{q}, 1 \omega+1 \omega_{n}\right)\right\},
$$

where $\hat{\mathcal{G}}$ is the matrix Green's function in particle-hole and spin-space, where $\omega_{n}=2(n+1) \pi / \beta$ are fermionic Matsubara frequencies. In the static $(\omega=0)$ and uniform $(\mathbf{q}=0)$ limit, Eq. (26) reduces to the Knight shift $\kappa \equiv \chi(0,0)$. We define the normalized Knight shift as

$$
\frac{\kappa}{\kappa_{0}}=\frac{\beta}{8 \pi} \sum_{\sigma} \int_{0}^{2 \pi} \int_{-E_{F}-\sigma I M}^{\infty} \frac{\mathrm{d} \theta \mathrm{d} \varepsilon N_{\sigma}(\varepsilon)}{\cosh ^{2}\left[\beta E_{\sigma}(\varepsilon, \theta) / 2\right]} .
$$

The Knight shift is a measure of the polarizibility of the conduction electrons in the compound, and serves as a highly useful probe to distinguish between singlet and triplet superconductivity. For a singlet superconductor, the total spin $S$ of the Cooper pair is zero, and the Knight shift therefore vanishes at $T=0$ since there are no quasiparticle excitations in the superconductor that may be polarized. The Knight shift vanishes regardless of the direction in which the external magnetic is applied for a singlet superconductor. For a triplet superconductor, this is quite different. The Knight shift now may be anisotropic in terms of the direction in which the magnetic field is applied. By means of the $\mathbf{d}_{\mathbf{k}}$-vector formalism [see Eq. (1)], one may infer that the Knight shift is unaltered even for $T<T_{c}$ when $\mathbf{d}_{\mathbf{k}} \perp \mathbf{H}$, but is altered according to Eq. 27 when $\mathbf{d}_{\mathbf{k}} \| \mathbf{H}$. This is valid as long as the $\mathbf{d}_{\mathbf{k}}$ remains 'pinned' in the material due to e.g. spin-orbit coupling, and hence does not rotate with $\mathbf{H}$. Otherwise, the Knight shift would remain unaltered in any direction. Therefore, an anisotropic Knight shift is a strong signature of a vector character of the superconducting order parameter, and hence of a spin-triplet superconducting state.

In Fig. 5, we plot the Knight shift for several values of $\tilde{I}$. It is interesting to note that $\kappa(0)$ is reduced with increasing $\tilde{I}$. Physically, this may be understood by realizing that the density of states of ungapped minority spins at the Fermi level decreases as the exchange splitting between the majority- and minority bands increases. This results directly in a lower amount of polarizable quasiparticles, and hence the Knight shift becomes suppressed. For a fully polarized ferromagnet (half-metal), the Knight shift would therefore be identical to an $s$-wave singlet superconductor for an applied field satisfying $\mathbf{H} \| \mathbf{d}_{\mathbf{k}}$. This fact emphasizes the importance of measuring the spin susceptibility along several directions to identify the proper spin-symmetry of the superconductor.

As a final experimental probe for the interplay between ferromagnetism and superconductivity, we employ a BlonderTinkham-Klapwijk formalism ${ }^{29}$ to calculate the tunneling between a normal metal and a ferromagnetic superconductor in the clean limit, using the self-consistently obtained values of the order parameters in the problem. From the results of Ref. 42, we find that the normalized tunneling conductance may be written as

$$
\frac{G}{G_{0}}=\sum_{\sigma} \int_{-\pi / 2}^{\pi / 2} \mathrm{~d} \theta \cos \theta\left[1+\left|r_{\sigma}^{A}(e V, \theta)\right|^{2}-\left|r_{\sigma}^{N}(e V, \theta)\right|^{2}\right],
$$

where $G_{0}$ is the normal-state conductance. Above, $r_{\sigma}^{A}(e V, \theta)$ and $r_{\sigma}^{N}(e V, \theta)$ designate the Andreev- and normal-reflection coefficient, respectively, and read 


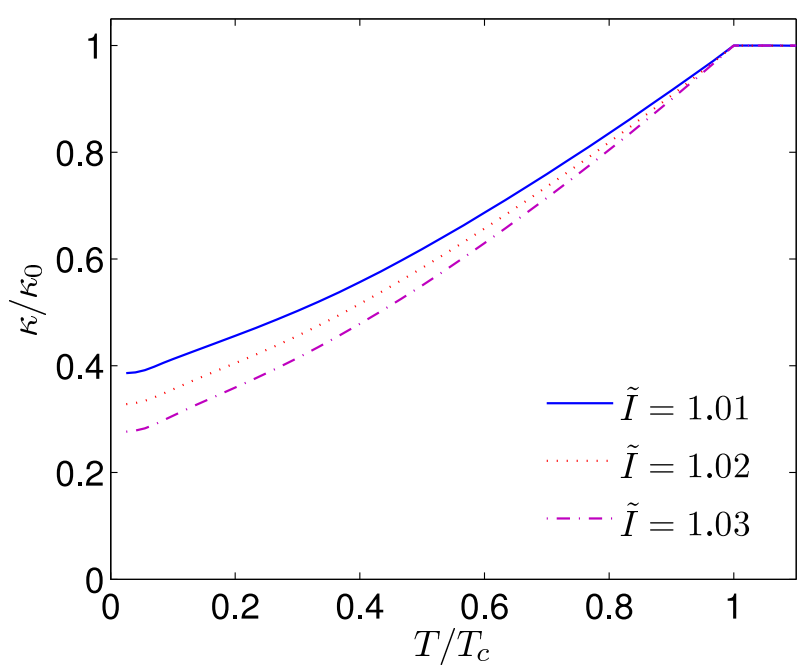

FIG. 5: (Color online) Knight shift for a ferromagnetic superconductor, using self-consistently obtained order parameters, for three different values of $\tilde{I}$. The field is here applied $\mathbf{H} \| \mathbf{d}_{\mathbf{k}}$.

$$
\begin{aligned}
r_{\sigma}^{N} & =-1+\frac{2 k_{F} \cos \theta\left[u_{\sigma}\left(\theta_{s+}^{\sigma}\right) u_{\sigma}\left(\theta_{s-}^{\sigma}\right)\left(\Upsilon_{+}^{\sigma}\right)^{*}+v_{\sigma}\left(\theta_{s-}^{\sigma}\right) v_{\sigma}\left(\theta_{s+}^{\sigma}\right) \gamma_{\sigma}\left(\theta_{s-}^{\sigma}\right) \gamma_{\sigma}^{*}\left(\theta_{s+}^{\sigma}\right)\left(\Upsilon_{-}^{\sigma}\right)^{*}\right]}{u_{\sigma}\left(\theta_{s+}^{\sigma}\right) u_{\sigma}\left(\theta_{s-}^{\sigma}\right)\left|\Upsilon_{+}^{\sigma}\right|^{2}-v_{\sigma}\left(\theta_{s-}^{\sigma}\right) v_{\sigma}\left(\theta_{s+}^{\sigma}\right) \gamma_{\sigma}\left(\theta_{s-}^{\sigma}\right) \gamma_{\sigma}^{*}\left(\theta_{s+}^{\sigma}\right)\left|\Upsilon_{-}^{\sigma}\right|^{2} .} \\
r_{\sigma}^{A} & =\frac{4 k_{F} \cos \theta q^{\sigma} \cos \theta_{s}^{\sigma} v_{\sigma}\left(\theta_{s+}^{\sigma}\right) u_{\sigma}\left(\theta_{s-}^{\sigma}\right) \gamma_{\sigma}^{*}\left(\theta_{s+}^{\sigma}\right)}{u_{\sigma}\left(\theta_{s+}^{\sigma}\right) u_{\sigma}\left(\theta_{s-}^{\sigma}\right)\left|\Upsilon_{+}^{\sigma}\right|^{2}-v_{\sigma}\left(\theta_{s-}^{\sigma}\right) v_{\sigma}\left(\theta_{s+}^{\sigma}\right) \gamma_{\sigma}\left(\theta_{s-}^{\sigma}\right) \gamma_{\sigma}^{*}\left(\theta_{s+}^{\sigma}\right)\left|\Upsilon_{-}^{\sigma}\right|^{2}} .
\end{aligned}
$$

We have defined $Z=2 m V_{0} / k_{\mathrm{F}}$ as a measure of the barrier strength, where $m$ is the quasiparticle mass, $V_{0}$ is the scattering strength of the barrier, and $k_{\mathrm{F}}$ is the Fermi momentum. Moreover, $\theta$ is the angle of incidence of incoming electrons from the normal side and we have implicitly incorporated conservation of group velocity and conservation of momentum parallel to the barrier, i.e. $k_{\mathrm{F}} \sin \theta=q^{\sigma} \sin \theta_{s}^{\sigma}$. Finally, we have introduced

$$
\Upsilon_{ \pm}^{\sigma}=q^{\sigma} \cos \theta_{s}^{\sigma} \pm k_{F} \cos \theta \pm{ }_{1} k_{F} Z
$$

and $\gamma_{\sigma}(\theta)=\Delta_{\sigma}(\theta) /\left|\Delta_{\sigma}(\theta)\right|, \theta_{s+}^{\sigma}=\theta_{s}^{\sigma}, \theta_{s-}^{\sigma}=\pi-\theta_{s}^{\sigma}$. In the quasiclassical approximation $E_{F} \gg\left(\Delta_{0}, \varepsilon\right)$, the wavevectors read

$$
k_{\mathrm{F}}=\sqrt{2 m E_{F}}, q^{\sigma}=\sqrt{2 m\left(E_{F}+\sigma I M\right)}
$$

while the spin-generalized coherence factors are

$$
\begin{aligned}
& u_{\sigma}\left(\theta_{s \pm}^{\sigma}\right)=\frac{1}{\sqrt{2}}\left\{1+\sqrt{1-\left(\left|\Delta_{\sigma}\left(\theta_{s \pm}^{\sigma}\right)\right| / E\right)^{2}}\right\}^{1 / 2}, \\
& v_{\sigma}\left(\theta_{s \pm}^{\sigma}\right)=\frac{1}{\sqrt{2}}\left\{1-\sqrt{1-\left(\left|\Delta_{\sigma}\left(\theta_{s \pm}^{\sigma}\right)\right| / E\right)^{2}}\right\}^{1 / 2} .
\end{aligned}
$$

In Fig. 6, we plot the conductance spectra of a nor$\mathrm{mal} /$ ferromagnetic superconductor junction. By writing the gap as $\Delta=\Delta_{0} \cos (\theta-\alpha)$, we allow for an arbitrary orientation of the gap with respect to the crystallographic axes. The features seen in the conductance spectra are qualitatively different for $\alpha=0$ and $\alpha=\pi / 2$. In the first case, the electronand hole-like quasiparticles entering the superconductor experience a constructive phase-interference which gives rise to the formation of a zero-energy state that is bound to the surface of the superconductor. The resonance condition for the formation of such zero-energy states is $\Delta(\theta)=-\Delta(\pi-\theta), \frac{53}{,}$ and the bound states are manifested as a giant peak in the zero-bias conductance ${ }^{54}$. Note that such states exist even if the spatial depletion of the superconducting order parameter is not taken into account, which may be shown analytically $\frac{56}{}$. Taking into account the reduction the gap experiences close to the interface compared to its bulk value, is known to yield the same qualitative features as the usual step-function approximation, with the exception of additional, smaller peaks at finite bias voltages due to non-zero bound states 57 . From Fig. 6, we see that the effect of increasing the exchange field amounts to sharper features in the conductance spectra. With increasing $\tilde{I}$, the zero-bias conductance peak becomes larger for $\alpha=0$, while the dip structure for $\alpha=\pi / 2$ becomes more pronounced. Physically, this may be understood by the increased contribution from majority spin carriers. The contribution from the minority spin carriers is constant for the entire low-energy regime, and leads to less pronounced features in the conductance. The effect of the barrier strength $Z$ is seen in the left column of Fig. 6 . For $\alpha=0$, increasing $Z$ leads to a higher peak at zero bias, while increasing $Z$ suppresses the 

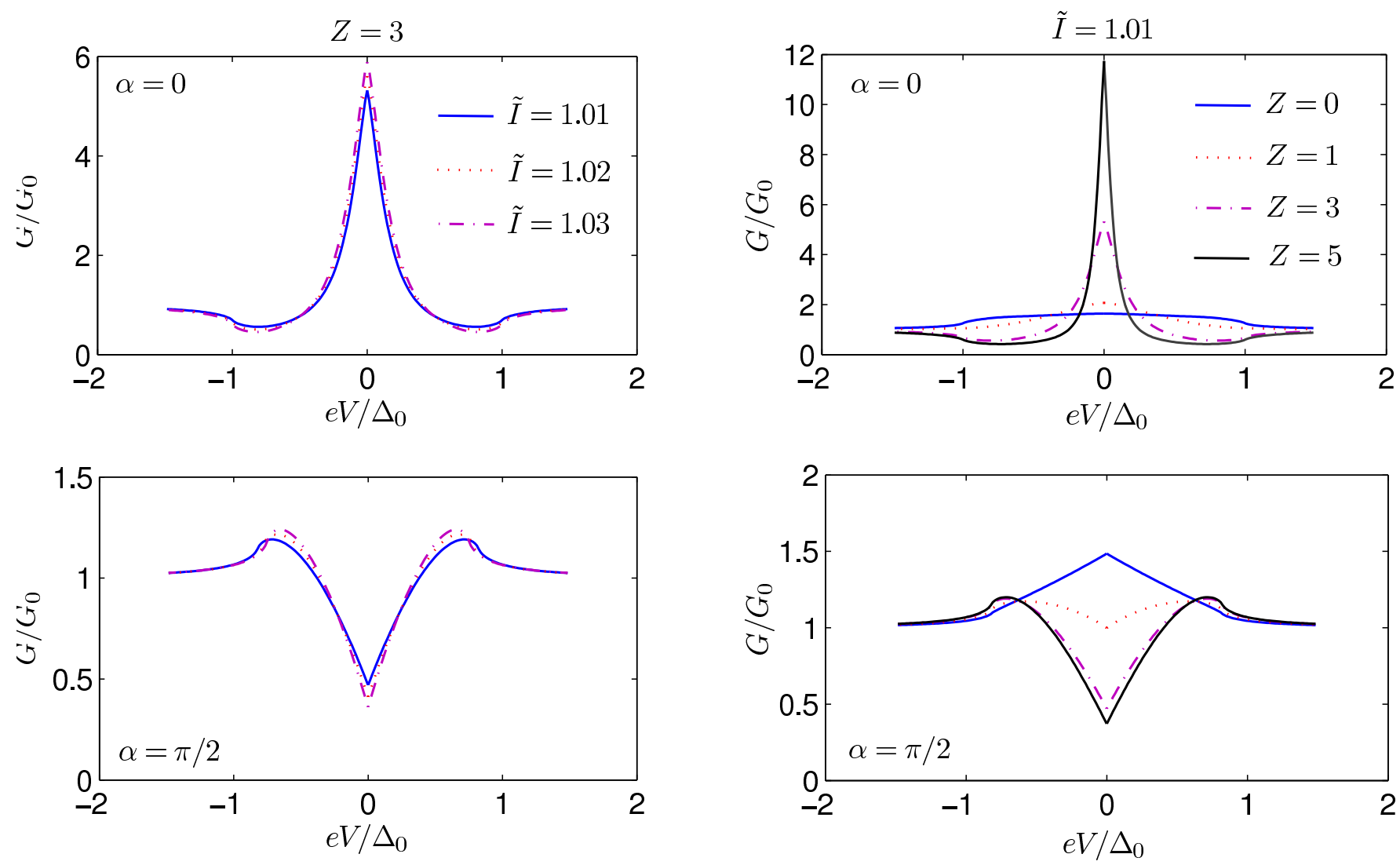

FIG. 6: (Color online) Plot of the tunneling conductance of a normal/ferromagnetic superconductor junction for $\alpha=0$ and $\alpha=\pi / 2$, using self-consistently obtained solutions at $T=0$. In the left column, we fix the tunneling barrier strength $Z=2 \mathrm{~m} V_{0} / k_{F}=3$ and plot the conductance for several values of the Stoner interaction $\tilde{I}$. In the right column, we fix $\tilde{I}=1.01$ and plot the conductance for several values of $Z$.

conductance for $\alpha=\pi / 2$.

It is also worth emphasizing the relation between the tunneling conductance and the bulk DOS of the superconductor. As is well-known, the conductance of a normal/s-wave superconductor junction in the tunneling limit approaches the DOS of the bulk superconductor ${ }^{29}$. The same argument is valid for a $d_{x^{2}-y^{2}}$-wave superconductor ${ }^{54}$. One might be tempted to conclude that the tunneling conductance will always approach the bulk DOS of the superconductor in the strong barrier limit as long as there is no formation of zero-energy states. However, closer examination reveals that this is not necessarily so.

To illustrate this, we draw upon some results obtained in Ref. 59. In general, the conductance of an N/S junction in the tunneling limit may be written as

$$
G(e V) \approx \frac{\int_{-\pi / 2}^{\pi / 2} \mathrm{~d} \theta_{N} \sigma_{N} \cos \theta_{N} \rho_{S}(e V)}{\int_{-\pi / 2}^{\pi / 2} \mathrm{~d} \theta_{N} \sigma_{N} \cos \theta_{N}}
$$

where $\sigma_{N}$ is the normal-state conductance for a given angle of incidence $\theta_{N}$ and $\rho_{S}$ is the surface DOS for the superconductor. In the absence of zero-energy states, the surface DOS coincides with the bulk DOS of the superconductor, i.e.
$\rho_{S}=\rho_{0}$, where

$$
\rho_{0}(e V)=\int_{-\pi / 2}^{\pi / 2} \mathrm{~d} \theta_{N} \operatorname{Re}\left\{\frac{e V}{\sqrt{e V^{2}-\left|\Delta\left(\theta_{N}\right)\right|^{2}}}\right\} .
$$

An important consequence of the above equation is that the tunneling conductance may be interpreted as the expectation value of $\rho_{S}$ with a weighting factor $\sigma_{N} \cos \theta_{N}$.

Let us now compare three different superconducting symmetries to illustrate the relation between the conductance and the DOS. We consider an $s$-wave, $d_{x^{2}-y^{2}}$-wave, and $p_{y}$-wave symmetry, none of which feature zero-energy surface states (Fig. 8). Naively, one might therefore expect that the conductance should converge towards $\rho_{0}$ in the tunneling limit. However, it turns out that the weighting factor $\sigma_{N} \cos \theta_{N}$, which is peaked around $\theta_{N}=0$, plays a major role in this scenario. In Fig. 7, we plot both the tunneling conductance $G(\mathrm{eV}) / G_{0}$ and the bulk DOS $\rho_{0}$ for these three symmetries and fix $Z=20$. We regain the well-known results that $G(\mathrm{eV}) / G_{0} \rightarrow \rho_{0}$ for large $Z$ in the $s$-wave and $d_{x^{2}-y^{2-}}$ case. However, the conductance and DOS differ in the $p_{y^{-}}$ wave case.

The reason for the deviation between $G / G_{0}$ and $\rho_{0}$ in the $p_{y}$-wave case may be understood by consulting Fig. 8 As seen, the weighting factor is peaked around normal incidence 

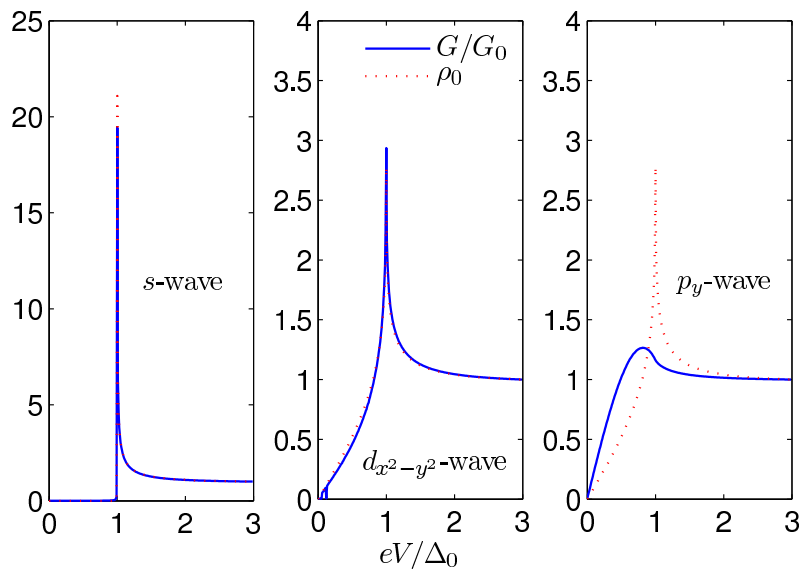

FIG. 7: (Color online) Plot of the normalized conductance $G / G_{0}$ and bulk DOS $\rho_{0}$ for three different symmetries of the superconducting state in the tunneling limit. Only in the $p_{y}$-wave case is there a difference between these two quantities.
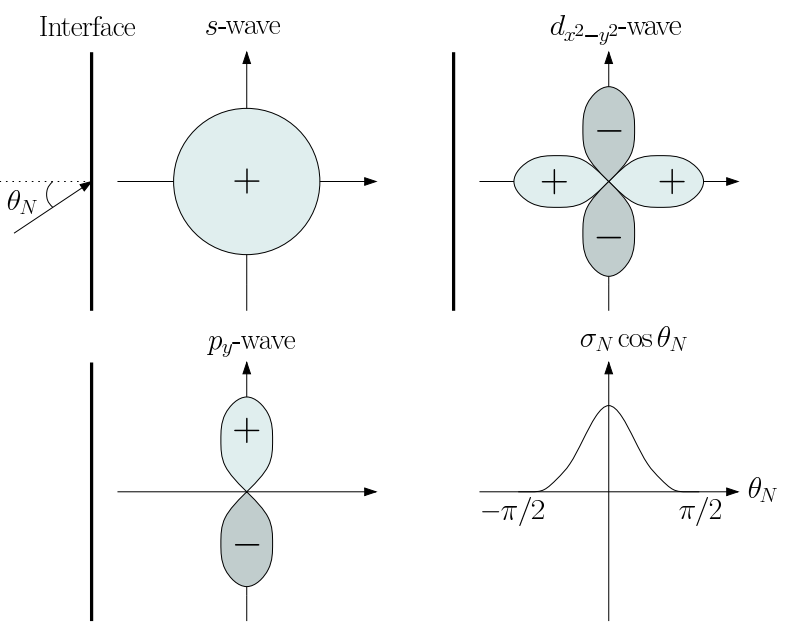

FIG. 8: (Color online) Illustration of the different symmetry states considered here and a qualitative sketch of the weighting factor $\sigma_{N} \cos \theta_{N}$.

$\theta_{N}=0$. In the $s$-wave and $d_{x^{2}-y^{2}}$-case, the gap magnitude is maximal at $\theta_{N}=0$ and replacing the weighting factor in Eq. (33) with unity has little or no consequence. The situation is dramatically different in the $p_{y}$-wave case. Now, the gap magnitude is actually zero for normal incidence, and it is precisely this contribution that will dominate the integration over angles in Eq. (33). Therefore, replacing the weighting factor with unity, in order to obtain the DOS, has a non-trivial consequence in the $p_{y}$-wave case. This analysis illustrates how the conductance and bulk DOS in the absence of zero-energy states are not always the same in the tunneling limit. Note that the orientation of the interface with respect to the symmetry of the order parameter is crucial with regard to the measured conductance spectra and the surface DOS. For instance, even at $\alpha=\pi / 4$ there is an appearance of a large zero-bias conductance peak for the $p$-wave pairing considered here, although the gap orientation does not satisfy the condition for perfect formation of zero-energy states.

\section{DISCUSSION}

We have discussed a mean-field model where itinerant ferromagnetism coexists with non-unitary, triplet superconductivity, with a gap that contains line nodes. The precise symmetry of the order parameter in the ferromagnetic superconductors $\mathrm{UGe}_{2}$, URhGe, UCoGe is still under debate, although most experimental findings and theoretical considerations strongly point towards the realization of a triplet superconducting order parameter. It is plausible that such a superconducting order parameter is non-unitary, thus breaking time-reversal symmetry in the spin channel of the Cooper pair.

The orbital symmetry of the superconducting order parameter in ferromagnetic superconductors is a more subtle issue. In Ref. 26, a mean-field model for isotropic, chiral $p$-wave gaps in a background of itinerant ferromagnetism was constructed. In that work, pairing was assumed to occur both for majorityand minority-spins, resulting in for instance a double-jump structure in the specific heat capacity. An isotropic, chiral $p$-wave order parameter has a constant magnitude, which is favorable in terms of maximizing the condensation energy gained in the superconducting state. Assuming an isotropic density of states at the Fermi level and a separable pairing potential of the form $V_{\mathbf{k k}^{\prime}}=-g \lambda_{\mathbf{k}} \lambda_{\mathbf{k}^{\prime}}$, the condensation energy gained at $T=0$ in the superconducting state reads

$$
E=-\frac{N(0) \Delta_{0}^{2}}{2}\left\langle\left|\lambda_{\mathbf{k}}\right|^{2}\right\rangle
$$

where $\Delta_{0}$ is the maximum value of the gap and $\langle\ldots\rangle$ denotes the angular average over the Fermi surface. This clearly shows the advantage of an isotropic gap $\left|\lambda_{\mathbf{k}}\right|=1$. The general principle is well-known: the system prefers to have the Fermi surface as gapped as possible. However, factors such as spinorbit pinning energy and lattice structure may conspire to prevent a fully isotropic gap. We also note that in our model, the ferromagnetic ordering enters at a much higher temperature than the superconducting order unless $\tilde{I}$ is very close to unity. This is consistent ${ }^{1,2}$ with the experimental findings for the ratio between the critical temperatures for ferromagnetic and superconducting order, $T_{c}^{\mathrm{FM}} / T_{c}^{\mathrm{SC}}$, except for $\mathrm{UCoGe}$ where the ratio is $\simeq 3.4$

The experiments performed so far are indicative of a single gap, or at least a strongly suppressed second gap, in the ferromagnetic superconductors. For instance, no double-jump features have been observed in the specific heat capacity ${ }^{1}$ for $\mathrm{UGe}_{2}$. This warrants the investigation of a single-gap model, possibly with line nodes as suggested by Harada et al..$^{20}$ Theoretically, the absence of the SC gap in the minority spin subband can be justified by considering the effect of Zeeman splitting on the electronic density of states (see discussion in Sec. [II] and Ref. 25). In general, it should be possible to discern the presence of two gaps by analyzing specific heat or point-contact spectroscopy measurements, unless one of the gaps is very small. 
Apart from this, another possible scenario, specific to $\mathrm{UGe}_{2}$, can be invoked to explain the observed gapless behaviour in the minority spin subband. This is the metamagnetic transition that occurs inside the FM phase of $\mathrm{UGe}_{2}$ and separates the two ferromagnetic phases with different values ${ }^{60,61}$ of magnetization $M$. The reason this meta-magnetic transition in $\mathrm{UGe}_{2}$ is of great importance is because the specific heat measurements clearly indicate ${ }^{60}$ that the maximum of superconducting $T_{c}$ occurs not at the FM to PM transition, but at some lower pressure $p_{x} \approx 12$ kbar that coincides precisely with the meta-magnetic transition ${ }^{20,61}$.

One can think of this transition as a point where the value of low-temperature magnetization $M$ sustains a jump. While the microscopic origin of this transition is not known, an idea has been put forward ${ }^{62}$ that it may be due to a sharp change in the density of states (DOS) due to the existence of a double peak in its structure close to the Fermi level. What happens according to this scenario is that applied pressure makes the Fermi level "sweep through" the double-peak structure in the DOS, thereby sharply increasing the density of states in the majority spin channel. It follows from a simple Stoner instability argument that such an increase in the DOS would lead to a larger value of effective interaction $\tilde{I} \equiv I N(0)$ and thus higher magnetization $M$. But this also means that the ratio of the DOS in the two spin channels, $N_{\uparrow} / N_{\downarrow}$, sharply increases at the meta-magnetic transition. It follows from Eqs. 16, 18, 22) that the ratio between the SC gaps in the two spin subbands

$$
\frac{\Delta_{\downarrow}}{\Delta_{\uparrow}} \propto \frac{T_{c}^{\downarrow}}{T_{c}^{\uparrow}}=\frac{\exp \left(-1 / g N_{\downarrow}\right)}{\exp \left(-1 / g N_{\uparrow}\right)}
$$

thus becomes very small, justifying the assumption $\Delta_{\downarrow}=0$ made in this work.

We note in passing that from an experimental point of view, a complication with $\mathrm{UGe}_{2}$ is that the superconductivity does not appear at ambient pressure, in contrast to URhGe and UCoGe. The necessity of considerable pressure restricts the use of certain experimental techniques, and this is clearly a challenge in terms of measuring for instance conductance spectra of $\mathrm{UGe}_{2}$. Another experimental quantity which would be of high interest to obtain from for instance $a b$ initio calculations, is the thermal expansion coefficient, which may be directly probed in high-pressure experiments 58 .

We also underline that in our model the magnetism is assumed to coexist uniformly with superconductivity. Depending on the geometry of the sample, it is likely that the intrinsic magnetization gives rise to a self-induced vortex phase. In a thin-film structure where the thickness $t$ is smaller than the vortex radius $\lambda$, we expect that ferromagnetism and superconductivity may be realized in a vortex-free phase, similarly to a thin-film $s$-wave superconductor in the presence of an inplane magnetic field. Further refinements leading to a more realistic model of a ferromagnetic superconductor should include the presence of spin-orbit coupling, which inevitably is present in heavy-fermion superconductors, in addition to the presence of vortices. Nevertheless, we believe that our model should capture important qualitative features of how the interplay between ferromagnetism and superconductivity may be manifested in experimentally accessible quantities. In particular, experiments on transport properties of ferromagnetic superconductors, such as the Josephson current and pointcontact spectroscopy, would be of high interest to further illucidate the pairing symmetry realized in ferromagnetic superconductors.

\section{SUMMARY}

In conclusion, we have constructed a mean-field theory of triplet superconductivity in the background of itinerant ferromagnetism, where the superconducting order parameter contains line nodes and the minority spin band remains ungapped at the Fermi level. We have solved the self-consistency equations for the order parameters in the problem, and find that ferromagnetism enhances superconductivity, while the ferromagnetism itself is virtually unaffected by the presence of superconductivity. We have made several predictions for experimentally accessible quantities: heat capacity, Knight shift, and tunneling conductance spectra. Our results may be helpful in the interpretation of experimental data, and could provide tools concerning the issue of identifying the pairing symmetry of ferromagnetic superconductors.

\section{Acknowledgments}

J.L. wishes to express his gratitude to Y. Tanaka at Nagoya University for his hospitality, where parts of this work were completed. N. Sato is also thanked for very useful discussions. This work was supported by the Norwegian Research Council Grants No. 158518/431 and No. 158547/431 (NANOMAT), and Grant No. 167498/V30 (STORFORSK).
${ }^{1}$ S. S. Saxena , P. Agarwal, K. Ahilan, F. M. Grosche, R. K. W. Haselwimmer, M. J. Steiner, E. Pugh, I. R. Walker, S. R. Julian, P. Monthoux, G. G. Lonzarich, A. Huxley, I. Sheikin, D. Braithwaite, and J. Flouquet, Nature 406, 587 (2000).

${ }^{2}$ D. Aoki, A. Huxley, E. Ressouche, D. Braithwaite, J. Flouquet, J.-P. Brison, E. Lhotel, and C. Paulsen, Nature 413, 613 (2001).

3 C. Pfleiderer, M. Uhlarz, S. M. Hayden, R. Vollmer, H. v. Löhneysen, N. R. Bernhoeft, and G. G. Lonzarich, Nature (Lon- don) 412, 58 (2001).

${ }^{4}$ N.T. Huy, A. Gasparini, D.E. de Nijs, Y. Huang, J.C.P. Klaasse, T. Gortenmulder, A. de Visser, A. Hamann, T. Gorlach, and H. v. Lohneysen, Phys. Rev. Lett. 99, 067006 (2007).

5 Tetsuya Ohta, Yusuke Nakai, Yoshihiko Ihara, Kenji Ishida, Kazuhiko Deguchi, Noriaki K. Sato, Isamu Satoh, arXiv:0712.1403.

${ }^{6}$ E.A. Yelland, S.M. Hayden, S.J.C.Yates, C. Pfleiderer, M. Uhlarz, 
R. Vollmer, H.v. Lohneysen, N.R. Bernhoeft, R.P. Smith, S. S. Saxena, and N. Kimura, Phys. Rev. B 72, 214523 (2005).

7 A. A. Abrikosov and L. P. Gorkov, Zh. Eksp. Teor. Fiz. 39, 1781 (1960) [Sov. Phys. JETP 12, 1243 (1961)].

8 A. M. Clogston, Phys. Rev. Lett. 9, 266 (1962).

${ }^{9}$ B. S. Chandrasekhar, Appl. Phys. Lett. 1, 7 (1962).

${ }^{10}$ P. Fulde and R. A. Ferrel, Phys. Rev. 135, A50 (1964); A. I. Larkin and Y. N. Ovchinnikov, Zh. Eksp. Teor. Fiz. 47, 1136 (1964).

11 D. Fay and J. Appel, Phys. Rev. B 22, 3173 (1980).

12 Ironically, Fay and Appe $1^{11}$ have developed their theory with $\mathrm{ZrZn}_{2}$ as a potential application in mind, but it took further 20 years before superconductivity was finally discovered in this compound ${ }^{3}$.

13 S. Tsutsui, M. Nakada, M. Saeki, S. Nasu, Y. Haga, E. Yamamoto, and Y. Onuki, Phys. Rev. B 60, 37 (1999).

${ }^{14}$ N. I. Karchev, K. B. Blagoev, K. S. Bedell, and P. B. Littlewood, Phys. Rev. Lett. 86, 846 (2001).

15 R. Shen, Z. M. Zheng, S. Liu, and D. Y. Xing, Phys. Rev. B 67, 024514 (2003).

${ }^{16}$ M. Cuoco, P. Gentile, and C. Noce, Phys. Rev. Lett. 91, 197003 (2003).

17 Although the superconductivity is observed at ambient pressure in $\mathrm{URhGe}^{2}, \mathrm{UCoGe}^{4}$ and $\mathrm{ZrZn}_{2}{ }^{\frac{3}{}}$, it is believed that it occurs on the border of ferromagnetism and that one could in principle suppress the Curie temperature to zero by applying pressure (sometimes a negative pressure would be required), making these compounds similar in this respect to $\mathrm{UGe}_{2}$, where $\mathrm{SC}$ is observed ${ }^{1,3}$ in the vicinity of the pressure-tuned phase transition.

18 J. A. Hertz, Phys. Rev. B 14, 1165 (1976); A. J. Millis, Phys. Rev. B 48, 7183 (1993).

19 A. J. Leggett, Rev. Mod. Phys. 47, 331 (1975).

20 A. Harada, S. Kawasaki, H. Mukuda, Y. Kitaoka, Y. Haga, E. Yamamoto, Y. Onuki, K. M. Itoh, E. E. Haller, H. Harima, Phys. Rev. B 75, 140502 (2007).

21 A. A. Abrikosov and L. P. Gor'kov, Zh. Eksp. Teor. Fiz. 39, 1781 [Sov. Phys. JETP 12, 1243 (1961)].

22 T. Ohmi and K. Machida, Phys. Rev. Lett. 71, 625 (1993).

${ }^{23}$ K. Machida and T. Ohmi, Phys. Rev. Lett. 86, 850 (2001).

${ }^{24}$ K. V. Samokhin and M. B. Walker, Phys. Rev. B 66, 174501 (2002); K. V. Samokhin and M. B. Walker, Phys. Rev. B 66, 024512 (2002); M. B. Walker and K. V. Samokhin, Phys. Rev. Lett. 88, 207001 (2002).

25 A. H. Nevidomskyy, Phys. Rev. Lett. 94, 097003 (2005).

26 J. Linder and A. Sudbø, Phys. Rev. B 76, 054511 (2007).

27 Notice that time-reversal symmetry may be spontaneously broken in the orbital part (angular momentum) of the Cooper pair wavefunction even if the state is unitary.

${ }^{28}$ F. Hardy and A. D. Huxley, Phys. Rev. Lett. 94, 247006 (2005).

${ }^{29}$ G. E. Blonder, M. Tinkham, and T. M. Klapwijk, Phys. Rev. B 25, 4515 (1982).

30 A. B. Shick and W. E. Pickett, Phys. Rev. Lett. 86, 300 (2001)

31 J. R. Schrieffer, Superconductivity, 1st ed. (Benjamin/Cummings, Reading, 1964).

32 J. Shi and Q. Niu, cond-mat/0601531

${ }^{33}$ H. Kotegawa, A. Harada, S. Kawasaki, Y. Kawasaki, Y. Kitaoka, Y. Haga, E. Yamamoto, Y. Onuki, K. M. Itoh, and E. E. Haller, J.
Phys. Soc. Jpn. 74, 705 (2005).

34 S. Tewari, D. Belitz, T. R. Kirkpatrick, and J. Toner, Phys. Rev. Lett. 93, 177002 (2004).

35 V. P. Mineev, cond-mat/0507572.

${ }^{36}$ V. P. Mineev and K. V. Samokhin, Introduction to Unconventional Superconductivity (Gordon and Breach, New York, 1999).

37 D. V. Shopova and D. I. Uzunov, Phys. Rev. B 72, 024531 (2005).

${ }^{38}$ M. L. Kulic, C. R. Physique 7, 4 (2006); M. L. Kulic, and I. M. Kulic, Phys. Rev. B 63, 104503 (2001).

39 I. Eremin, F. S. Nogueira, and R.-J. Tarento, Phys. Rev. B 73, 054507 (2006).

${ }^{40}$ M. S. Grønsleth, J. Linder, J.-M. Børven, and A. Sudbø, Phys. Rev. Lett. 97, 147002 (2006).

41 J. Linder, M. S. Grønsleth, and A. Sudbø, Phys. Rev. B 75, 024508 (2007).

42 J. Linder, M. S. Grønsleth, and A. Sudbø, Phys. Rev. B 75, 054518 (2007).

43 T. Yokoyama and Y. Tanaka, Phys. Rev. B 75, 132503 (2007).

44 A. Brataas and Y. Tserkovnyak, Phys. Rev. Lett. 93, 087201 (2004).

45 Y. Zhao and R. Shen, Phys. Rev. B 73, 214511 (2006).

46 A. G. Lebed, Phys. Rev. B 59, R721 (1999); A. G. Lebed, K. Machida, and M. Ozaki, Phys. Rev. B 62, R795 (2000).

${ }^{47}$ K. Sengupta, I. Žutic, H.-J. Kwon, V. M. Yakovenko, and S. Das Sarma, Phys. Rev. B 63, 144531 (2001)

48 C. J. Bolech and T. Giamarchi, Phys. Rev. Lett. 92, 127001 (2004); C. J. Bolech and T. Giamarchi, Phys. Rev. B 71, 024517 (2005).

49 R. D. Duncan, C. D. Vaccarella, and C. A. S de Melo, Phys. Rev. B 64, 172503 (2001).

50 J. Linder, A. Nevidomskyy, and A. Sudbø, unpublished.

51 C. Bernhard, J. L. Tallon, E. Brcher, and R. K. Kreme, Phys. Rev. B 61, R14960 (2000); J. L. Tallon, J. W. Loram, G. V. Williams, and C. Bernhard, Phys. Rev. B 61, R6471 (2000); A. Fainstein, E. Winkler, A. Butera, and J. Tallon, Phys. Rev. B 60, R12597 (1999).

52 M. Sigrist, AIP Conference Proceedings 789, 165 (2006).

53 C.-R. Hu, Phys. Rev. Lett. 72, 1526 (1994).

${ }^{54}$ Y. Tanaka and S. Kashiwaya, Phys. Rev. Lett. 74, 3451 (1995).

55 E. P. Wohlfarth, Phys. Lett. 75A, 141 (1979).

56 T. Lofwander, V. S. Shumeiko, and G. Wendin, Supercond. Sci. Technol. 14, R53 (2001).

57 Y. S. Barash, A. A. Svidzinsky, and H. Burkhardt, Phys. Rev. B 55, 15282 (1997).

58 N. Sato, private communication.

59 S. Kashiwaya, Y. Tanaka, M. Koyanagi, and K. Kajimura, Phys. Rev. B 53, 2667 (1996).

${ }^{60}$ N. Tateiwa, T. C. Kobayashi, K. Hanazono, K. Amaya, Y. Haga, R. Settai, and Y. Onuki, J. Phys. Condens. Matter 13, L17 (2001); N. Tateiwa, T. C. Kobayashi, K. Amaya, Y. Haga, R. Settai, and Y. Onuki, Phys. Rev. B 69, 180513(R) (2004).

${ }^{61}$ M. Uhlarz, C. Pfleiderer, and S. M. Hayden, Phys. Rev. Lett. 93, 256404 (2004).

${ }^{62}$ K. G. Sandeman, G. G. Lonzarich, and A. J. Schofield, Phys. Rev. Lett. 90, 167005 (2003). 